\title{
DÉCLENCHEMENT DE L'CESTRUS ET OBTENTION PRÉCOCE DE GESTATIONS CHEZ DES AGNELLES A L'AIDE D'ÉPONGES VAGINALES IMPRÉGNÉES D'ACÉTATE DE FLUOROGESTONE ( $\left.{ }^{1}\right)$
}

\author{
J. THIMONIER, P. MALLÉON, Y. COGNIÉ, R. ORTAVANT \\ avec la collaboration technique de $\mathrm{C}$. CoRNu \\ Centre de Recherches vétérinaires et zootechniques, \\ Physiologie de la Reproduction 37 - Nouzilly \\ Institut national de la Recherche agronomique
}

\section{SOMMAIRE}

La possibilité de provoquer l'œestrus et l'ovulation et d'obtenir la gestation par traitement hormonal chez des agnelles prépubères de race Lacaune et chez des agnelles de race Ile-de-France âgées de 9 à I 3 mois, a été étudiée à différents moments de l'année.

L'injection de PMSG à la fin du traitement progestatif administré par voie vaginale (acétate de fluorogestone), se révèle indispensable pour obtenir un pourcentage élevé de venues en a’strus quelle que soit la période de l'année.

Le pourcentage de fertilité après traitement progestatif et injection de PMSG est voisin de $70 \mathrm{p}$. Ioo au début et en fin de saison sexuelle. Il est supérieur à celui des agnelles recevant le progestagène seul. Il diminue au fur et à mesure que l'on s'éloigne de la saison sexuelle. Il augmente avec le poids des animaux.

Les œstrogènes administrés en même temps que le progestagène, l'injection d'HCG à la fin du traitement, diminuent le pourcentage d'agnelles en ostrus et la fertilité.

\section{INTRODUCTION}

Si l'ovulation peut être obtenue chez des agnelles âgées de I à 2 mois (MANSOUR, I959; MaUrÉoN, non publié), le déclenchement du comportement d'œstrus semble lié à l'âge: 27 p. Ioo des agnelles traitées avec la progestérone et PMSG viennent en œstrus à l'âge de 5 mois et 75 p. IOo à l'âge de 8 mois (SEFIDBAKHT, FOOTE et MADSEN, I967). L'apparition normale du premier oestrus, qui caractérise

(1) Acétate de fluorogestone $=\mathrm{FGA}={ }_{17} \alpha$ acétoxyg $\alpha$ fluorom $\beta$ hydroxyprogestérone $=$ S. C. 9880. I'roduit Searle. 
la puberté, est en effet précédée par des cycles ovariens, chez les ovins (AI, IEN, I959; Sefidbakht, Madseis et Foote, ig66).

L'hypophyse d'agnelles prépubères est, comme celle des autres espèces, riche en hormones gonadotropes (MoORE, I965-66, Corbin et Daniels, 1967). L'action des stéroïdes ovariens peut permettre une facilitation de la décharge endogène de ces hormones et par là même, l'ovulation et l'apparition de l'œestrus. Le rôle de la progestérone sur le déclenchement de ce premier œestrus a effectivement été démontré (MAssotr, 1959).

Cependant, l'influence de la saison et du poids des agnelles sur le déclenchement hormonal de l'œestrus n'a pas été étudiée. Or, ces facteurs modifient l'âge à la puberté (HAMmoxi), I944; HAFE2, I952; WILLIAMS, I954; AI.IEN, I959; LANG et Highr, I 967 ).

Dans deux cas correspondants à des besoins précis de l'élevage ovin en France (production d'agneaux de boucherie-production de lait), nous avons étudié les facteurs qui influent sur l'induction de l'ostrus et l'obtention de la gestation chez les agnelles au voisinage de la puberté.

\section{MA'IÉRIEL E'T MF́'THODE}

\section{Animaux}

Des animaux appartcnant à deux races ovines ont été utilisés dans leur milieu d'originc.

a) Race Lacaune.

Les expérimentations ont été effectuées pendant deux années consécutives sur des troupeaux laitiers de la région des Causses. Ln 1965 , toutes les agnelles ont été traitées pendant la deuxième quiizaine du mois d'août. En I966, les traitements se sont étalés depuis la deuxième quinzaine du mois de juillet à la fin du mois daaût.

Jans cette région ( $44^{\circ}$ lat. Nord), les agnelles sont en général pubères aux mois de septembrew tobre, à l'âge de 9 à io mois et pour un poids vif de 45 à 50 kilogrammes.

La lut te pratiquée pendant ces 2 mois permet d'obtenir 40 ì 80 p. roo d'animaux gestants suivant les élevages. Leur lactation débutant en février-mars est écourtée par les éleveurs, par suite de la fermeture des laiteries dès le mois de juillet.

Nous avons cherché à obtenir une lutte plus précoce en juillet-août, sur la totalité des agnelles qui sont alors âgées de 7 a 8 mois. La période de lutte des agnelles serait ainsi très proche de celle des brebis adultes (juin-juillet).

\section{b) Race Ile-de-France.}

Les quatre expérimentations sur animaux de race Ile-de-France ont eu lieu dans le même élevage en janvier, mars et décembre Ig66, c'est-à-dire en fin de saison sexuelle ou en début de période d'anostrus, sur des animaux de i a à I 3 mois, et en juin ${ }_{1} 967$, c'est-à-dire en début de saison sexuelle. Dans ce cas, le's agnelles étaient alors âgées de 9 mois en moyenne.

Les animaux sont traditionnellement élevés en bergerie, dans le Bassin parisien (latitude $49^{\circ}$ Nord), et sont destinés à la production d'agneaux de boucherie. En général, un seul agnelage principal par an est pratiqué par les éleveurs. Les mise bas ont lieu soit au printemps soit à l'automne, suivant les élevages.

Les jeunes femelles sont la plupart du temps mises à la lutte à l'âge de 18 mois. Cependant, les animaux nés à l'automne sont parfois accouplés aux mois de juillet-août de l'année suivante: le pourcentage de mise bas est environ de $50 \mathrm{p}$. Iо०. 


\section{Traitements}

a) Race Lacaune.

La technique des éponges vaginales imprégnées de progestagène (RoBinson, 1965), nous a permis d'entreprendre facilement l'étude des différents facteurs intervenant lors du déclenchement hormonal de l'ostrus et sur la gestation chez les agnelles. Différentes doses de progestagène (FGA) déposées sur des éponges vaginales ont été essayées. Le tableau I indique les traitements auxquels ont été soumis les animaux et les nombres d'agnelles recevant chacun de ces traitements.

\section{TABLEAU I}

Agnelles de race Lacaune. Traitements et répartition des animaux recevant les traitements. Mois de juillet et août

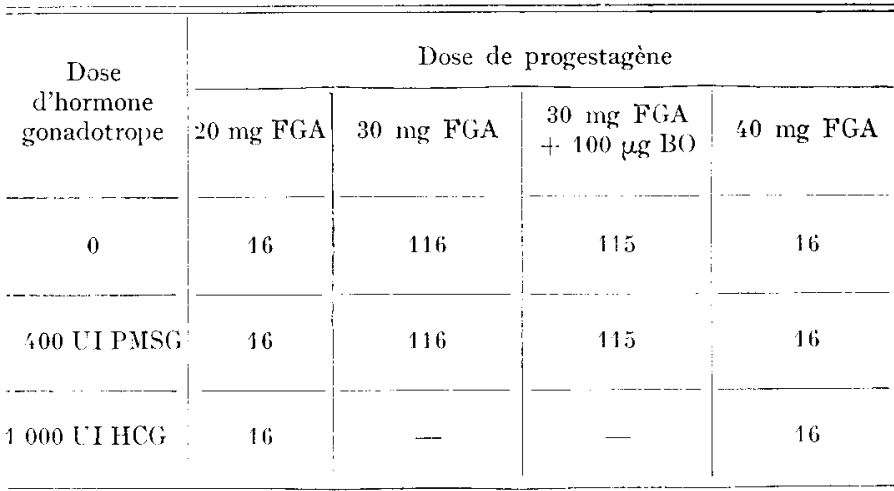

270 agnelles ont servi de témoins. Ejles n'ont pas reçu de placebo sans progestagène.

TABLEAU 2

Agnelles de race Ile-de-France. Traitements, époques de traitement et nombre d'animaux recevant ces traitements

\begin{tabular}{|c|c|c|}
\hline \multirow{2}{*}{$\begin{array}{l}\text { Dose d'hormone } \\
\text { gonadotrope }\end{array}$} & \multicolumn{2}{|c|}{ Dose de progestagène } \\
\hline & $30 \mathrm{mg} \mathrm{FGA}$ & 10 ol1 /5 mg FGA \\
\hline 0 & 25 (janvier) & 12 (mars) \\
\hline '00 UI PMSG & $\cdots-$ & $\begin{array}{l}36 \text { (décembre) } \\
68 \text { (juin) }\end{array}$ \\
\hline 600 UI PMSG & $\because 5$ (janvier) & $\begin{array}{l}12 \text { (mars) } \\
36 \text { (décembre) }\end{array}$ \\
\hline
\end{tabular}

112 agnelles ont servi de témoins mais n'ont pas reçu de placebo sans progestagène. 
La durée du traitement par progestagène a été de i 7 jours pour tous les animaux. L'hormone PMSG a été injectée par voie intramusculaire o ou $\mathbf{~} 2$ heures après la fin du traitement progestatif ; I oo $\mu \mathrm{g}$ de benzoate d'œestradiol ont été déposés sur une partie des éponges vaginales.

Les animaux ayant perdu leur éponge vaginale ont été systématiquement éliminés des calculs.

b) Race Ile-de-France.

Le même progestagène a été employé. Trois durées de traitement ont été inises à l'épreuve: I4, I6, I 7 jours. Les injections de PMSG ont été effectuées lors du retrait de l'éponge vaginale. Le tableau 2 indique les traitements, leur époque et le nombre d'animaux intéressés.

\section{Détection de l'ostrus}

La détection des chaleurs a été effectuée à l'aide de béliers vasectomisés ou entiers munis de tabliers, deux fois par jour, pendant les 5 jours suivant la fin du traitement, puis I fois par jour pendant 20 jours. Cependant, le contrôle de l'œestrus n'a pas pu être effectué en juin I 967 .

La lutte a eu lieu uniquement pendant cette période de 25 jours aussi bien pour les animaux traités que pour les animaux témoins. La plupart des agnelles ont été saillies naturellement. Quelques-unes seulement ont été inséminées artificiellement (race Lacaune).

\section{RÉSULTATS}

\section{Déclenchement de l'cestrus (tabl. 3)}

a) Infuence de $P M S G$.

Le traitement des agnelles à l'aide du progestagène permet une légère augmentation du pourcentage des animaux en œestrus. En début de saison sexuelle, avec les

TABLEAU 3

Pourcentages d'agnelles en oestrus après traitements progestatif et progestatif $+P M S G$. Races Lacaune et Ile-de-France

\begin{tabular}{|c|c|c|c|c|c|c|}
\hline \multirow[b]{2}{*}{ Race } & \multirow[b]{2}{*}{ Dose PMSG } & \multicolumn{4}{|c|}{ Dose progestagène } & \multirow{2}{*}{$\begin{array}{c}\text { Total } \\
A+\mathrm{B}+\mathrm{C}\end{array}$} \\
\hline & & 0 & $\begin{array}{c}20 \\
\mathrm{~A}\end{array}$ & $\begin{array}{c}30 \\
B\end{array}$ & $\begin{array}{c}40 \text { oul } 45 \\
\text { C }\end{array}$ & \\
\hline \multirow{2}{*}{ Lacaune } & 0 & 50,7 & 75,0 & 63,8 & 53,3 & 64,0 \\
\hline & $400 \mathrm{UI}$ & - & 100,0 & $97,1^{* *}$ & $100,0^{* *}$ & $97,8 * *$ \\
\hline \multirow{2}{*}{$\begin{array}{l}\text { Mle-de-france } \\
\text { (janvier-mars) }\end{array}$} & 0 & 15,9 & 一 & $\geq 9,2$ & 16,7 & 25,0 \\
\hline & $600 \mathrm{UI}$ & - & 一 & $64,0^{*}$ & $75,0^{*}$ & $67,6^{* *}$ \\
\hline
\end{tabular}

Les pourcentages ont été calculés à partir des nombres d'animaux ayant conservé leur éponge vaginale.

Déclenchement de l'œestrus :

Différences $0-{ }_{1}^{\prime} 00$ UI PMSG : $\left\{\begin{array}{l}* \text { significatives } \mathrm{P} \leqslant 0,05, \\ * * \text { hautement significatives } \mathrm{P} \leqslant 0,01 .\end{array}\right.$ 
animaux cle race Lacaune, 64,0 p. Ioo des agnelles traitées avec le progestagène présentent des chaleurs contre 50,7 p. Ioo des agnelles témoins.

Le même effet est observé en début de période d'anœstrus, ou en anœstrus, avec les animaux de race Ile-de-France: 25 , I p. Ioo des agnelles traitées ont un comportement d'œestrus contre $15,9 \mathrm{p}$. roo des agnelles témoins.

L'augmentation de la dose de progestagène diminue légèrement le pourcentage de venues en œstrus. Cette diminution n'est cependant pas significative.

Cette influence de la dose de progestagène disparaît complètement après injection de PMSG qui permet d'obtenir un pourcentage élevé d'agnelles en œstrus: Lacaune: 97,8 p. Ioo en début de saison sexuelle ; Ile-de-France: 67,6 p. 1oo pendant la période d'ancestrus.

Une bonne synchronisation est obtenue à la suite du traitement progestatifPMSG, quelle que soit la race (fig. I et 2), puisque 95 à Ioo p. Ioo des animaux sont en chaleurs 48 heures après le retrait de l'éponge vaginale.

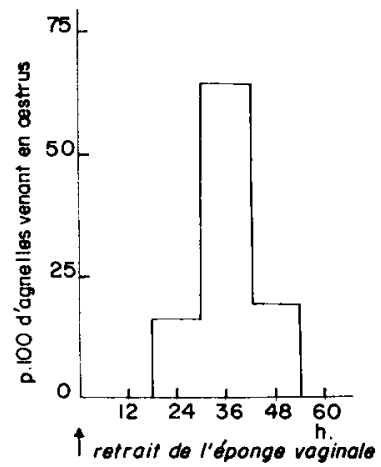

FIG. I. - Apparition de l'asirus après traltement progestalif + PMSG Agnelles de race Lacaune

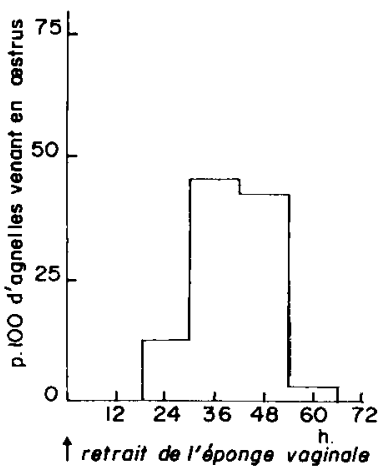

FIG. 2. - Apparition de l'ostrus après traitement progestatif $+P M S G$ Agnelles de race lle-de-France

b) Influence des cestrogènes et de HCG (tab1. 4).

TABLEAU 4. - Influence des astrogènes administris par soie vaginale en même temps que le progestagène, et de $11 C G$ injectée 12 heures après la fin du traitement progestatif sur l'apparition de l'astrus ches des agnelles de race Lacaune.

Pourcentage d'agnelles en a'strus après ces traitements

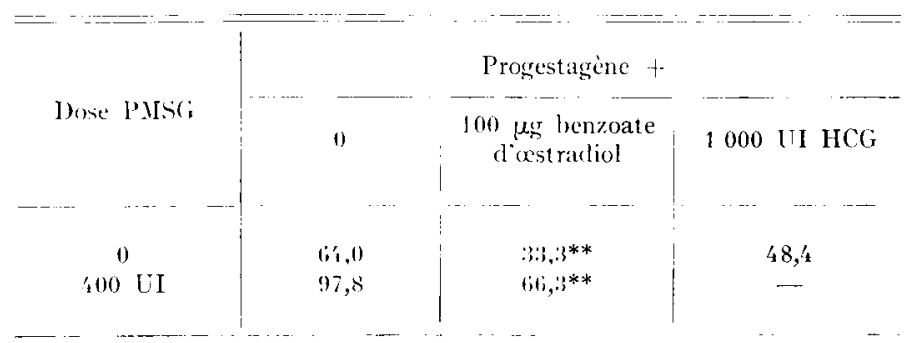

Les pourcentages ont été calculés ì partir des nombres d'animaux ayant conservé leur éponge vaginale.

** Différences entre traitements (progestarìne et progrestagène + - ostrogènes) hautement significatives : $\mathrm{P} \leqslant 0,01$. 
Les cestrogènes administrés en même temps que le progestagène, de même que 1'injection de $\mathrm{HCG}$, ont un effet défavorable sur l'apparition de l'œstrus : 33,3 p. Ioo et 48,4 p. Ioo respectivement des animaux recevant roo $\mu \mathrm{g}$ de benzoate d'œstradiol ou I ooo UI de HCG, viennent en œstrus contre 64,0 p. Ioo des agnelles traitées avec le progestagène seul. Les œestrogènes diminuent également le pourcentage d'apparition d'œstrus après traitement progestatif-PMSG.

c) Influence du moment de l'année (tabl. 5).

TABLEAU 5. - Influence de la période de traitement sur le déclenchement de l'astrus après traitement associant progestagène et $P M S G$ Races Lacaune et Ile-de-lrance

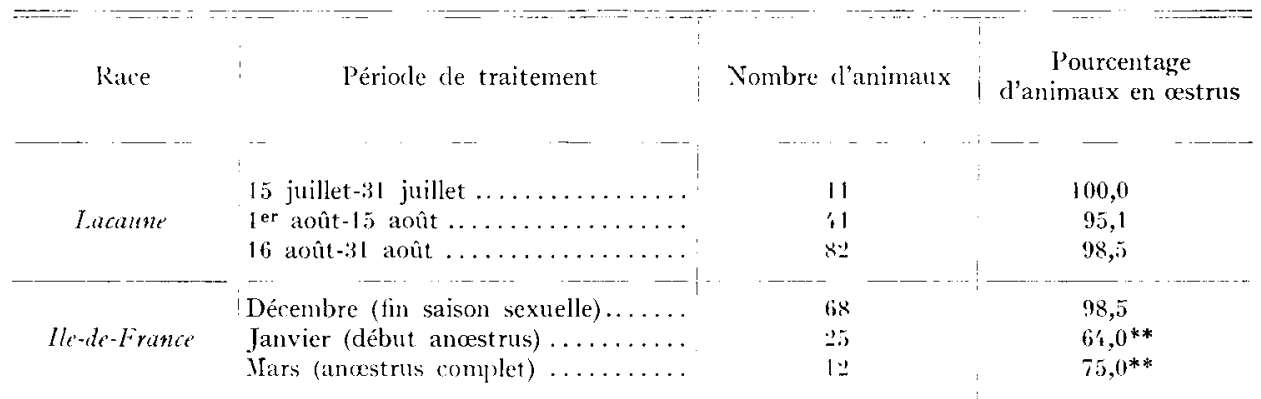

** Differences entre pourcentage d'oestrus du mois de lécembre et pourcentage d'oustrus des mois de janvier et mars, hatutenent sionificatives: $P^{3} \leqslant 0,01$.

İn début de saison sexuelle, après traitement progestatif-PMSG, le pourcentage d'agnelles de race Lacaune en oestrus ne varie pratiquement pas quelle que soit la période de traitement et reste tıès élevé : 95 à Ioo p. Ioo.

Le pourcentage d'animaux non gestants revenant en cestrus est faible ; 25.7 p. I 00 pour l'ensemble des animaux traités. Il n'atteint que 6 p. 100 chez les animaux témoins.

Mais l'influence du moment de l'année sur le déclenchement de l'œstrus apparaît nettement chez les agnelles de race Ile-de-France, même lorsque PMSG est utilisée : 98,5 p. Ioo des animaux sont en ostrus lorsque le traitement a lieu en fin de saison sexuelle, 64,0 et 75,0 p. Ioo s'il est effectué respectivement au début et au milieu de l'anœestrus saisonnier.

I e pourcentage d'animaux non gestants revenant en chaleur en fin de saison sexuelle atteint $1_{4}, 3$ p. Ioo chez les agnelles traitées, mais il reste nul chez les témoins. Pendant la période d'anoestrus, il est nul aussi bien chez les animaux témoins que chez les animaux traités.

d) Influence du poids des animaux (tab1. 6).

Chez les agnelles Lacaune, pour l'ensemble des animaux traités, l'influence du poids est importante : $58,3 \mathrm{p}$. Ioo seulement des animaux pesant de 30,5 à $40 \mathrm{~kg}$, viennent en cestrus contre $75,5 \mathrm{p}$. Ioo de ceux pesant plus de $40 \mathrm{~kg}$. Cependant, $1 \mathrm{e}$ traitement avec PMSG annule cette influence. 
TABLEAU 6

Influence du poids sur le déclenchement de l'trstrus Agnelles de race lacaune

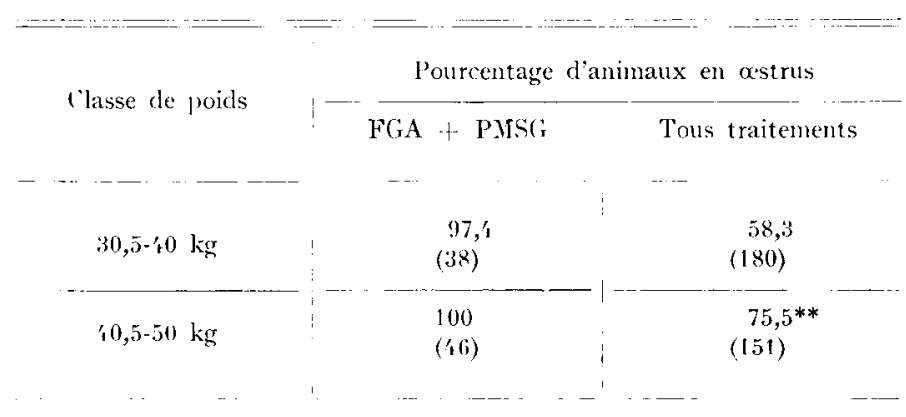

** Différence hautement significative cutre les pourcentages d'iestrus des 2 classes de poids : $P^{2} \leqslant 0,01$.

( ) Nombre d'animanx.

\section{Fertilité}

a) Définitions.

Le pourcentage de mise bas est le nombre moyen d'agnelles mettant bas pour Ioo agnelles saillies. Dans nos conditions expérimentales, le nombre des agnelles saillies est égal à celui des animaux en chaleurs. Nous ne considérerons que le pourcentage de mise bas à la chaleur suivant immédiatement le traitement.

Le pourcentage de fertilité est le nombre moyen d'agnelles mettant bas pour Ioo agnelles traitées. Il représente une résultante des pourcentages de mise bas et de venues en ostrus.

Nous calculerons le pourcentage de fertilité après le premier cestrus ou œstrus induit ( 5 jours de lutte après l'arrêt du traitement progestatif) et le pourcentage de fertilité pour toute la durée de l'expérimentation, c'est-à-dire pour une période de 25 jours après la fin du traitement progestatif. Les gestations établies lors d'un retour en cestrus après une première saillie non fécondante sont alors prises en considération.

Pour les animaux témoins, le pourcentage de mise bas est calculé en tenant compte uniquement du premier ostrus apparu pendant la période de 25 jours de lutte. Il en est de même pour le pourcentage de fertilité après le premier cestrus. Les gestations établies lors d'un retour en œstrus après une première saillie non fécondante interviennent dans le calcul du pourcentage de fertilité pour une période de 25 jours de lutte.

b) Infuence de PMSG (tabl. 7).

Les doses de progestagène sont sans influence sur les pourcentages de mise bas et de fertilité. A une époque de l'année, pour les 2 races, les animaux traités recevant 
ou non une injection intramusculaire de PMSG ont un pourcentage de mise bas identique à celui des animaux témoins. Ainsi, ce pourcentage est voisin de $55 \mathrm{p}$. Ioo

\section{TABLEAU 7}

Pourcentage de fertiliti après l'a'strus induil ches les agnelles des races Lacaune et Ile-de-France. Influence des traitements

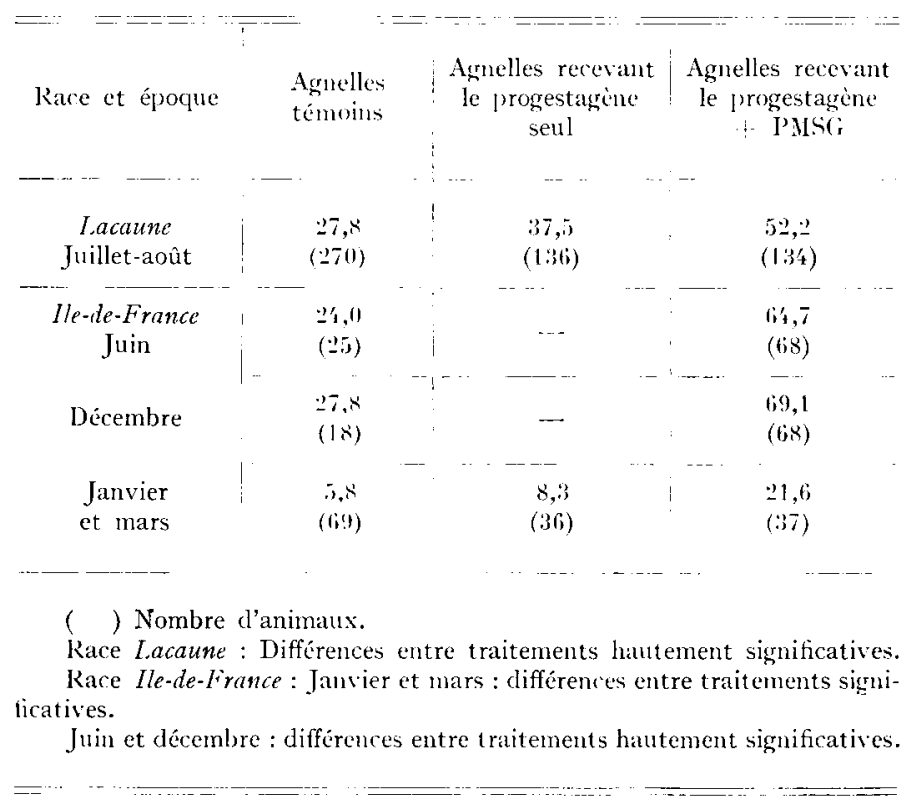

(entre 53,4 p. Ioo et 58,6 p. IOo) pour les agnelles de race Lacaune en début de saison sexuelle. Il est de $70 \mathrm{p}$. Ioo en fin de saison sexuelle et de $34 \mathrm{p}$. Ioo $(32,0$ à 36,4 p. IOo) en début d'ancestrus ou en anœestrus, pour les agnelles de race Ile-de-France.

Par contre, le traitement progestatif améliore pour les agnelles de race Lacaune, le pourcentage de fertilité après le premier ostrus et l'hormone PMSG l'augmente encore : 52,2 p. Ioo en race Lacaune et 2 I,6 p. Ioo en race Ile-de-France, contre respectivement $27,8 \mathrm{p}$. I oo et 5,8 p. Ioo pour les animaux témoins. Il s'agit d'un effet indirect lié à l'augmentation du pourcentage d'agnelles venant en œstrus à la suite du traitement (tabl. 3).

Le pourcentage de fertilité après 2 œstrus consécutifs (25 jours de lutte) n'est que très peu supérieur au pourcentage de fertilité après l'œestrus induit.

Ces deux pourcentages sont identiques en période d'ancestrus chez les agnelles Ile-de-France (tabl. 9). Les retours en ostrus peu nombreux chez les animaux non gestants après une première saillie ont donc un rôle négligeable dans l'amélioration du pourcentage de fertilité générale. 
c) Influence des cestrogènes et $H C G$ (tabl. 8).

Les cestrogènes et $\mathrm{HCG}$ ont un effet dépressif sur le pourcentage de mise bas : respectivement 34,4 p. I00 et 20 p. Ioo des agnelles venues en œestrus après un traitement associant progestagène et œestrogène ou $\mathrm{HCG}$ mettent bas, au lieu de 58,6 p. Ioo chez les animaux recevant le progestagène seul. L'hormone PMSG injectée à la fin du traitement progestatif-œestrogènes n'améliore pas ce pourcentage de mise bas $(37.7$ p. IOO).

TABIEAU 8

Influence des a'strogènes administrés par noie vaginale en même temps que le progestagène et de $H C G$ sur le pourcentage de mise bas. Agnelles de race Jacaune

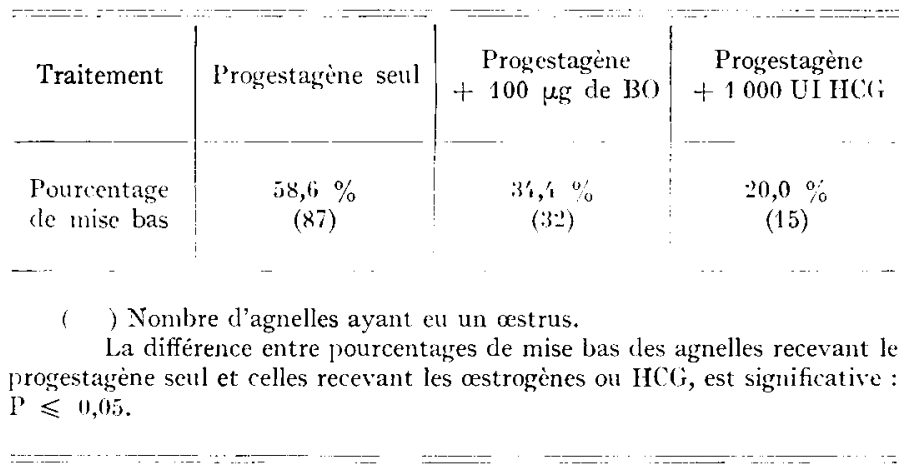

d) Infuence du moment de l'année ou de l'âge (tab1. 9).

Chez les agnelles de race Lacaune recevant PMSG, en début de saison sexuelle, le pourcentage de mise bas augmente au fur et à mesure que l'on se rapproche du milieu de la saison sexuelle. Il passe de 9 , I p. Ioo pendant la deuxième quinzaine du mois de juillet, à $4 \mathrm{I}, 0$ p. Ioo pendant le début đu mois d'août pour atteindre 68,0 p.IOO durant la deuxième moitié du même mois.

Le pourcentage de fertilité après 5 jours de lutte suit d'ailleurs une progression identique pendant la même période.

Chez les agnelles de race Ile-de-France, avant le début de la saison sexuelle normale, le pourcentage de fertilité après 5 jours de lutte est élevé $(64,7 \mathrm{p}$. 100). I1 en est de même en fin de saison sexuelle (69, I p. roo). Le passage de la saison sexuelle à la période d'ancestrus se traduit par une brusque diminution de ce pourcentage (2I,6 p. IOO), bien que les animaux soient plus âgés.

L,e moment de l'année influe sur le pourcentage de fertilité, non seulement parce que l'œestrus est plus difficile à induire chez les agnelles en période d'anœestrus, mais aussi parce que la gestation s'établit plus difficilement à la suite de cet œestrus. 


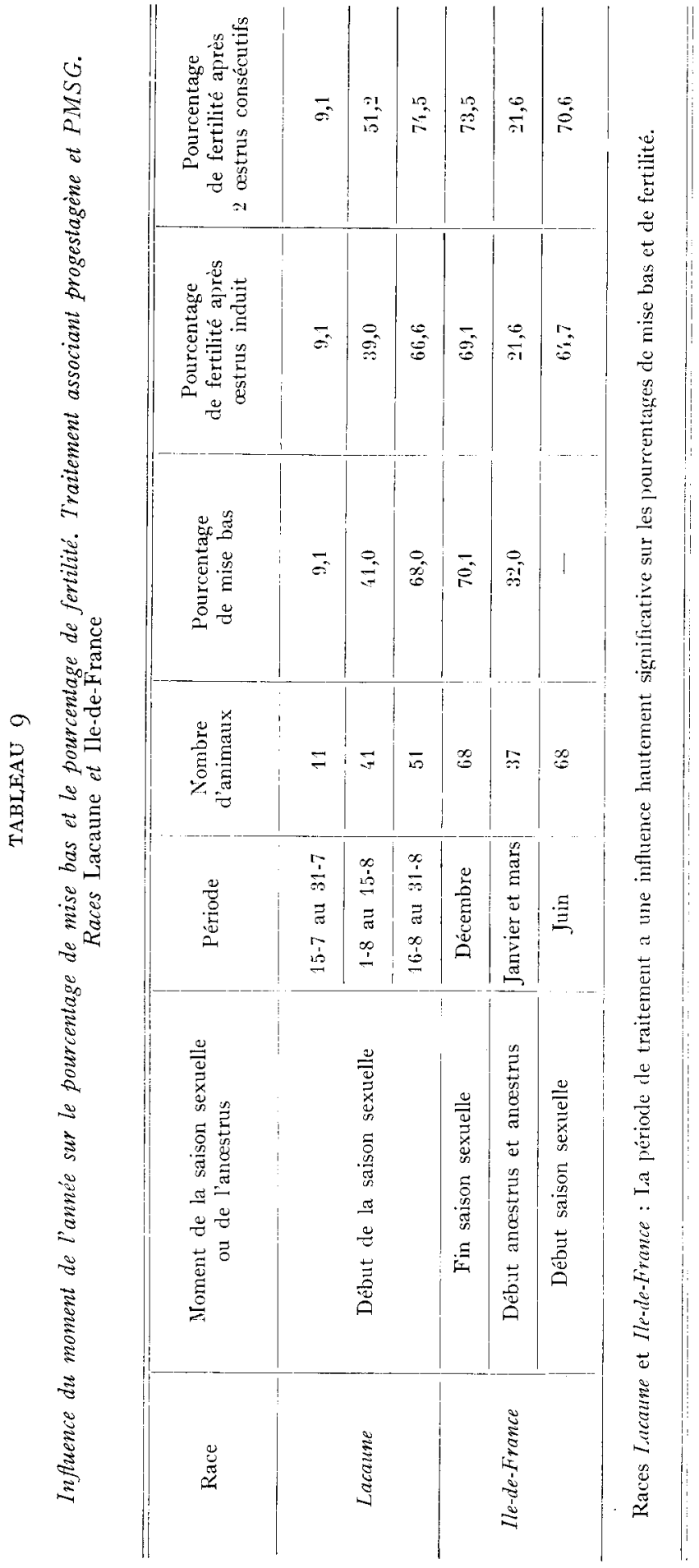


e) Influence du poids des animaux (tabl. Io).

Le poids vif des animaux a une influence importante sur le pourcentage de mise bas : ce pourcentage augmente avec le poids des animaux. Les pourcentages de fertilité subissent la même influence.

TABLEAU IO

Inthence du poids des animaux sur les pourcentages de mise bas et fertilité Traitement progestatif +400 UI PMGS. Race Lacaune

\begin{tabular}{c|c|c|c|c}
\hline $\begin{array}{c}\text { Classe } \\
\text { de poids }\end{array}$ & $\begin{array}{c}\text { Nombre } \\
\text { d'animaux }\end{array}$ & $\begin{array}{c}\text { Pourcentage } \\
\text { de mise bas }\end{array}$ & $\begin{array}{c}\text { Pourcentage } \\
\text { de fertilité } \\
\text { après ostrus } \\
\text { induit }\end{array}$ & $\begin{array}{c}\text { Pourcentage } \\
\text { de fertilité } \\
\text { après 2 ostrus } \\
\text { consécutifs }\end{array}$ \\
$30,5-40 \mathrm{~kg}$ & 38 & 48,6 & $47, \mathrm{t}$ & 55,3 \\
\hline $40,5-50 \mathrm{~kg}$ & 16 & 65,2 & 65,2 & $78,3^{*}$ \\
\hline
\end{tabular}

* Différence significative ențre les pourcentages de fertilité des 2 clisses de poids : $P \leqslant 0,05$.

\section{DISCUSSION}

\section{a) Obtention de l'xestrus}

Le déclenchement des chaleurs chez les agnelles peut donc être obtenu avec succès vers 1'âge de $7-8$ mois, chez les animaux de race Lacaune, et de 9 à 12 mois chez les agnelles de race Ile-de-France, à l'aide d'un traitement avec des progestagènes administrés par voie vaginale. Nos résultats sont comparables à ceux obtenus par Manunta et Casu (I967) : 85 p. Ioo des agnelles de race Sarde âgées de Io à I 2 mois traitées pendant la saison sexuelle, ont un œstrus induit après traitement progestatif par voie vaginale.

Une injection de PMSG est indispensable pour avoir la quasi-totalité des agnelles en cestrus, lorsque le traitement a lieu en début ou en fin de saison sexuelle.

On sait que PMSG facilite la décharge des hormones gonadotropes de l'hypophyse de rattes impubères (MCCormack et MEYER, I962 ; ZARrow et QUINN, I963; Brown-Grant, Quinn et Zarrow, rg64; WAGNER et BroWN-GraNt, 1965). I1 est vraisemblable qu'elle joue un rôle identique chez les agnelles dont on avance ainsi la puberté, puisque celle-ci semble liée à la libération des facteurs de décharge des gonadotropines (CORBIN et DANIELS, I967).

Les difficultés rencontrées pour induire l'œestrus en période d'anœstrus même chez des agnelles plus âgées, proviennent certainement de variations saisonnières des contenus hypophysaires en FSH et LH identiques à celles observées chez les animaux adultes ('Thimonier et Mauiśon, ig68). 
L’impossibilité de rendre cycliques ces jeunes animaux en début ou en fin de la période qui correspond à la saison sexuelle des adultes, confirme que la saison sexuelle des agnelles est plus courte que celle des adultes (HAFEZ, I952; MILIER, Ig62).

Les cestrogènes ajoutés sur l'éponge vaginale contenant le progestagène diminuent le pourcentage d'agnelles venant en ostrus. Chez le Rat, l'administration simultanée de mestranol et de noréthynodrel bloque la synthèse de $\mathrm{L} / \mathrm{H}$ et diminue la décharge de FSH (LABSHETWAR, I967). Le nombre de corps jaunes obtenus après un tel traitement est beaucoup plus faible qu'après un traitement de mestranol ou de noréthynodrel seul. Un effet identique peut être supposé dans nos expériences.

\section{b) Obtention de la gestation}

L'effet défavorable observé sur le pourcentage de mise bas avec le progestagène chez les animaux adultes, pendant la saison sexuelle (FOORD, I966 ; ROBERTS, I966; RobINSON et LAMOND, Ig66; WISHART, I966-I967) n'apparaît pas chez les agnelles. Ce pourcentage atteint en effet 70 p. Ioo.

Pour un même moment de l'année, ce pourcentage est identique chez les agnelles témoins et traitées avec ou sans supplémentation PMSG.

Mais ce pourcentage de mise bas varie avec l'âge et le poids des agnelles au moment du traitement et avec la saison; le bélier peut être responsable en partie de la variation saisonnière. La diminution avec l'âge et le poids peut provenir soit de la mauvaise qualité des gamètes prođuits, soit de difficultés d'implantation.

Les aufs provenant d'une ovulation provoquée même chez les animaux de 30 jours sont fécondables (Mauríon et Thibaurt, non publié). Chez des antenaises, 69 à 88 p. Ioo des oufs récupérés sont fécondés (GORDON, I958). Nous ne connaissons pas le pourcentage de fécondation chez les agnelles âgées de 8 à 12 mois. Il est vraisemblable qu'il est du même or đre. La survie des cufs fécondés en fonction de 1 'âge des animaux est inconnue.

Pour des animaux de même âge, le niveau nutritionnel a une influence sur le poids de l'utérus (ALLEN, I959). Les échecs d'implantation chez des agnelles de poids faible peuvent probablement être attribués au sous-développement de l'utérus.

Mais l'absence d'implantation peut provenir aussi d'un déséquilibre cestrogènesprogestérone qui dépend du développement $d u$ corps jaune, des décharges hypophysaires en FSH, LH et LTH. L'hypophyse des animaux de poids faible à un âge donné est-elle moins chargée en ces hormones? AILEN (1959) apprécie l'activité globale gonadotrope par le test du testicule de poulet. Il conclut à l'absence de variation en contenu gonadotrope des hypophyses d'agnelles ayant des taux de croissance différents. Chez les brebis adultes, Howland et al. (Ig66) montrent qu'un. niveau nutritionnel faible entraîne une diminution de la concentration hypophysaire en LH, mais n'a pas d'effet sur la concentration hypophysaire en FSH. PIAcsek et MeITes (I967) observent une diminution de la décharge de FSH et LH ainsi qu'tune diminution de la synthèse de LH chez des rattes sous-alimentées. Chez ces animaux, l'endomètre utérin est dégénéré, indiquant un manque d'œstrogènes circulant.

Les taux de croissance différents ne sont pas toujours imputables aux différences de niveau nutritionnel. Cependant, les observations que nous avons faites dans les élevages nous font penser que le niveau nutritionnel est le facteur principal. 
L'influence de la saison sur le pourcentage de gestation peut donc se faire par l'intermédiaire des variations probables des teneurs hypophysaires, en FSH et LH chez les agnelles; les sécrétions des stéroïdes responsables de l'implantation subiraient par conséquent une variation saisonnière.

\title{
CONCLUSION
}

Pendant la saison sexuelle, utilisé par voie vaginale, l'acétate de fluorogestone en association avec PMSG donne une bonne synchronisation des chaleurs sur la totalité des agnelles prépubères. Pendant cette période, un pourcentage de fertilité supérieur à 70 est obtenu quelle que soit la race des animaux, mais dans certaines conditions d'âge et de poids. Dans ces conditions, le traitement donne un pourcentage de fertilité supérieur à celui des animaux non traités.

A u cours de l'ancestrus saisonnier, le déclenchement de l'oestrus est plus difficile et le pourcentage de fertilité est plus faible. Pour cette période, les conditions d'âge et de poids restent à déterminer avant d'utiliser la technique des éponges vaginales chez les jeunes animaux.

Reçu pour publication en avril 1968.

\section{REMERCIEMEN'S}

Nous remercions le laboratoire de Zootechnie de l'École nationale supérieure agronomique de Montpellier qui a participé à toutes les expérimentations sur agnelles de race Lacaune, et la Confédération générale des Producteurs de Lait de Brebis et des Industriels de Roquefort, la Société des Caves de Roquefort, les éleveurs qui ont participé aux différentes expérimentations et la Société CLIN-BYLA qui a fourni l'acétate de fluorogestone nécessaire pour les expériences.

\section{SUMMARY}

\author{
INDUCTION OF GSTRUS AND EARLY PREGNANCY IN EWE-LAMBS \\ BI MEANS OF PESSARIES IMPREGNATED WITH FLUOROGESTONE ACETATE
}

Induction of cestrus and pregnancy by means of an intravaginal progestagen treatment (Robinson, 1965) was studied on 7 to 8 months old Lacaune and 9 to 13 months old Ile-de-France ewe-lamb at various times of the year.

PMSG supplementation is required for the induction of cestrus in 97 to Ioo per cent Lacaune ewe-lambs, whatewer their body weights, in July or August - which corresponds to the onset of the seasonal breeding activity of adult ewes. The supplementation is also required for Ile-de-France ewe-lambs at the end of the breeding season.

During the anocstrus period, only 64 to 75 per cent of progestagen-treated ewe-lambs came in cstrus, even with PMSG supplementation (tables $3,5,6$ ).

(Estrogens administered together with the progestagen treatment or IICG injection at the end of the treatment have an inhibitory effect on ocstrous response (table 4).

Fertility rate after progestagen-treatment increased until mid-breeding season. It ranged from 9 to 68 per cent for Lacaune ewe-lambs and from 32 to 70 per cent for Ile-de-France (table 9). It also increased with body weight, viz 47.4 per cent in ewe-lambs weighing 30 to $40 \mathrm{~kg}$ versus 65.2 per cent in ewe-lambs weighing more than $40 \mathrm{~kg}$.

The lambing rate (i.e. average number of lambs per roo mated ewes) also increased with body weight of ewe-lambs for a given time of year. It is identica! in control and in treated ewe-lambs wether or not PMSG-supplemented. 


\section{RÉFÉRENCES BIBLIOGRAPHIQUES}

AlLEN 1. X., 1959. Some factors affecting fertility in female sheep. Ph. D. Thesis, Universily of Nottingham.

BROWN-GRANT K., (UINX D. L., ZARROW IIX., 1964. Superovulation in the androgen-treated immature Rat. Endocrinology, 74, 81 I-813.

Corbin A., Daniels F. L., ig67. (hanges in concentration of female Rat pituitary Fill and staik nediane eminence follicle stimulating hormone releasing factor with age. Veurnentocinolog!', 2, 304-3I+.

Foord II. F., I 966 . Observations on the use of progesterone impregnated tampons in a herd of Dorsel Hill sheep. Vet. Rec., 78, 46I.

GORDON I., I958. The use of progesterone and serum gronadotrophin (P'.M.S) in the control of fertility in sheep. II Studies in the extraseasonal production of lambs. J.Agric. Sci., 50, $152-197$.

HaFez E. S. E., 1952. Studies on the breeding season and reproduction of the ewe. J. lgric. Soi., 42, $180-265$.

Hammoni) J. Jr., r944. (On the breeding season in the sheep. J. Agric. Sri., 34, 97-105.

IIOWtand B. E., Kirpatrick R. L., POlE A. L., CASIIn I.. T.., ig6o. Pituitary and ovarian function in ewes fed on two nutritional levels. J. Anim. Sci., 25, 7 $10-728$.

LABHSETwaR A. P., I 907 . Effects of norethynodrel and mestranol on pituitary LH in the fenale Rat. J. Reprod. Ferl., 14, 379-385.

Lavo; D. R., Hight ( $;.$ K., ig67. Age of puberty, lenght of breeding season and ovulation rate in Romme. Marsh and Border leicester $\times$ Rommey Marsh Hoggets. Proc. N. Z. Soc. A nim. Prod., 27. ti.

McCormack C. E., MeYer R. K., ig62. Ovulating hormone release in gonadotrophin treated immature rats. Proc. Soc. Exp. Biol. Med., 110, 343-346.

Mansour A. M., 1959. The hormonal control of ovulation in the immature lamb. Agric Sici.. 52, S9-94.

Manunta (i., Cast S., 1067. Sulla sincronizzazione dell'estro nelle annelle. Boll. Soc. Ital. Biol. Sper., 43, $508+5 \mathrm{II}$.

MILler III., W. W., 1962. Studies of ovarian activity and fertility in ewes and ewe lanbs. Ph. I. Thesis. Auburn Unizersily.

Moork W. W., Ig65-60. Changes in pituitary I.H concentration in prepubertal and postpubertal rats. Veuroendocrinology, 1, 333-340.

Piacser B. E., Meites J., 1967. Reinitiation of gonadotropin release in underfed Ratts by constant light or epinephrine. Endocrinology, 81, 535-5+1.

QIINA D.L., ZarRow MI. X., I964. Inhibition of pregnant mare's serum induced orulation in the immature Rat. Endocrinology, 74, 309-3 3 .

RoBERTS E. M., 1966. The use of intravaginal sponges impregnated with 6-nethy-1 7 -aretoxyprogesterone (MAP) to synchronize ovarian activity in cyclic Merino ewes. Proc, Iust. Soc. Hilm. Prod., 6, 32-37.

RoBinson 'T. J., I 965 . Use of progestagen-impregnated sponges inserted int ravaginally or subutaneously for the control of the oestrous cycle in the sheep. Nalne, 206, 39.

Robinson T. J., Lavoni, D. R., ig66. Control of reproduction in sheep and cattle. Proc. Arst. Soc. Anim. Prod., 6, 10-18.

Sefidbaknt N., Madsen M. A., Foote W. C., i 966 . P'uberal estrus and ovulation of lambs (Abst.) J. Anim. Sci., 25, 586.

Sefidrakit N., Foote W. C., Madske M. A., 1967. Iomonal induction of estrus and ovulation in prepuberal ewes (Abst.). J. Anim. Sci., 26, 95 I.

Thmonier J., Maulion P., 1968 . Variations saisomicres du comportement doestrus et des activites ovarienne et hypophysaire chez les Ovins. Ann. Biol. Anim. Bioch. Biophys. (sous presse).

WAgNer J. W., Brow-Ginat K., Ig65. Studies on the time of luteinizing hormone releatse in gonadotrophin treated immature Rat. Endocrinology', 76, $95^{8-065}$.

Williams S. M., 1954. Fertility in Clim foresl sheep. J. Agric. Sci., 54, 202.

WisinarT D. F., 1966 . The induction of earlier breeding altivity in sheep. A comparison between the use of vasectomised rams and the use of intravaginal pessaries inpregnated with a new progestin. Vet. Ret. 79, 356-358.

Wisilart D. F., I967. Synchronization of cestrus in sheep: the use of pessaries. V'et. Rec., 81, $276-287$.

ZARROW M. X., QUIN D. L., 1963. Superovulation in the immature rat following treatment with PYS alone and inhibition of PiIS-induced ovulation. J. endecr., 26, 18 - -188 . 